TRANSACTIONS OF THE

AMERICAN MATHEMATICAL SOCIETY

Volume 352, Number 7, Pages 3029-3043

S 0002-9947(00)02404-1

Article electronically published on March 15, 2000

\title{
INFLECTION POINTS AND TOPOLOGY OF SURFACES IN 4-SPACE
}

\author{
RONALDO ALVES GARCIA, DIRCE KIYOMI HAYASHIDA MOCHIDA, \\ MARIA DEL CARMEN ROMERO FUSTER, AND MARIA APARECIDA SOARES RUAS
}

\begin{abstract}
We consider asymptotic line fields on generic surfaces in 4-space and show that they are globally defined on locally convex surfaces, and their singularities are the inflection points of the surface. As a consequence of the generalized Poincaré-Hopf formula, we obtain some relations between the number of inflection points in a generic surface and its Euler number. In particular, it follows that any 2 -sphere, generically embedded as a locally convex surface in 4 -space, has at least 4 inflection points.
\end{abstract}

\section{INTRODUCTION}

E.A. Feldman [8] showed that the number of umbilic points of a generic compact surface $M$ in $\mathbb{R}^{3}$ is always greater than or equal to $2|\chi(M)|$. Now, since the inverse of the stereographic projection from $\mathbb{R}^{3}$ to $S^{3}$ takes the umbilic points of $M$ to the inflection points (in the sense of Little [11]) of its image in $S^{3} \subset \mathbb{R}^{4}$, it follows that the number, $N$, of inflection points of a generic compact orientable surface contained in $S^{3}$ satisfies the inequality

$$
N \geq 2|\chi(M)| \text {. }
$$

It is then natural to ask whether this result holds for any generic convex compact surface of $\mathbb{R}^{4}$, in the sense that it is contained in the boundary of its convex hull. We show that this is true for the more general class of generic locally convex compact surfaces in $\mathbb{R}^{4}$. This is done by using geometrical information on the surface provided by the study of the generic singularities of the height functions on $M$ made in [17, together with the analysis of the asymptotic direction fields, whose singularities are, precisely, the inflection points of $M$. An immediate consequence of this is that any 2-sphere generically embedded as a locally convex surface of $\mathbb{R}^{4}$ has at least 4 inflection points.

For the more general case, including the nonlocally convex compact surfaces, we must take into account certain generically isolated points that we call parabolic cusps of $M$. They are characterized by the fact that $M$ has a unique asymptotic direction at them (so, they are parabolic) and it is tangent to the curve of parabolic points. We prove that if $N$ represents the sum of the numbers of (imaginary type)

Received by the editors September 29, 1997 and, in revised form, June 15, 1998.

1991 Mathematics Subject Classification. Primary 58C27; Secondary 53A05.

Key words and phrases. Inflection point, height function, asymptotic direction.

Research of the first author was partially supported by CNPq and FUNAPE, Brazil.

Research of the third author was partially supported by DGCYT, grant no. PB96-0785.

Research of the fourth author was partially supported by CNPq, Brazil, grant \# 300066/88-0. 
inflection points and of parabolic cusps of a surface $M$ with no inflection points of real type, then

$$
N \geq 2\left|\chi\left(\bar{M}_{h}\right)\right|,
$$

where $M_{h}$ is the hyperbolic part of $M$. As a consequence we analyze the topological structure of the singular set of the generalized Gauss map of a generic surface without inflection points and parabolic cusps.

Examples illustrating the results are discussed in the last section.

\section{Previous Results}

Let $f$ be a smooth embedding of a compact surface $M$ into $\mathbb{R}^{4}$. Given a point $m \in M$, consider the unit circle in $T_{f(m)} f(M)$ parametrized by the angle $\theta \in$ $[0,2 \pi]$. Denote by $\gamma_{\theta}$ the curve obtained by intersecting $f(M)$ with the hyperplane at $f(m)$ composed of the direct sum of the normal plane $N_{f(m)} f(M)$ and the straight line in the tangent direction represented by $\theta$. Such a curve is called normal section of $f(M)$ in the direction $\theta$. The curvature vector $\eta(\theta)$ of $\gamma_{\theta}$ in $f(m)$ lies in $N_{f(m)} f(M)$. Varying $\theta$ from 0 to $2 \pi$, this vector describes an ellipse in $N_{f(m)} f(M)$, called the curvature ellipse of $f(M)$ at $f(m)$. This ellipse may degenerate onto a radial segment of a straight line, in which case $f(m)$ is known as an inflection point of the surface. This inflection point is of real type when $f(m)$ belongs to the curvature ellipse, and of imaginary type when it doesn't. An inflection point is flat when $f(m)$ is an end point of the curvature ellipse. The torsion $\tau_{\theta}$ of $\gamma_{\theta}$ at $f(m)$ is called the normal torsion of $f(M)$ in the direction $\theta$ at $f(m)$. A direction $\theta$ in $T_{f(m)} f(M)$ for which $\frac{\partial \eta}{\partial \theta}$ and $\eta(\theta)$ are parallel is an asymptotic direction.

The $2^{\text {nd }}$ fundamental form of $f(M)$ is characterized by two quadratic forms. Their functional coefficients will be denoted by $(a, b, c)$ and $(e, f, g)$ respectively. We then have the following functions associated to them:

$$
\begin{gathered}
\Delta(m)=\frac{1}{4} \operatorname{det}\left[\begin{array}{rrrr}
a & 2 b & c & o \\
e & 2 f & g & o \\
o & a & 2 b & c \\
o & e & 2 f & g
\end{array}\right](m), \\
k(m)=\left(a c-b^{2}+e g-f^{2}\right)(m) \quad \text { (Gaussian curvature of M) }
\end{gathered}
$$

and the matrix

$$
\alpha(m)=\left(\begin{array}{ccc}
a & b & c \\
e & f & g
\end{array}\right)(m)
$$

By identifying $f(m)$ with the origin of $N_{f(m)} f(M)$ it can be shown that (see [11]):

(a) $\Delta(m)<0 \Rightarrow f(m)$ lies outside the curvature ellipse (such a point is said to be a hyperbolic point of $M)$.

(b) $\Delta(m)>0 \Rightarrow f(m)$ lies inside the curvature ellipse (elliptic point).

(c) $\Delta(m)=0 \Rightarrow f(m)$ lies on the curvature ellipse (parabolic point).

A more detailed study of case (c) allows us to distinguish among the following possibilities:

$\left(c_{1}\right) k(m)>0 \Rightarrow f(m)$ is an inflection point of imaginary type.

$\left(c_{2}\right) k(m)<0$ and $\left\{\begin{array}{l}\operatorname{rank} \alpha(m)=2 \Rightarrow \text { ellipse is nondegenerate, } \\ \operatorname{rank} \alpha(m)=1 \Rightarrow f(m) \text { is an inflection point of real type. }\end{array}\right.$ 
$\left(c_{3}\right) k(m)=0 \Rightarrow f(m)$ is an inflection point of flat type.

It can be shown that for an open and dense set of embeddings of $M$ in $\mathbb{R}^{4}, \Delta^{-1}(0)$ $\cap k^{-1}(0)=\phi$. It was proven in [14] that there are exactly two asymptotic directions at a hyperbolic point and just one asymptotic direction at a parabolic point, unless it is an inflection point, in which case all the directions are asymptotic.

In a previous paper [13] we characterized the different points of these surfaces by means of the singularities of the family of height functions on them as follows:

a) An elliptic point $f(m)(\Delta(m)>0)$ is a nondegenerate critical point for any of the height functions associated to normal directions to $f(M)$ at $f(m)$.

b) If $m$ is a hyperbolic point $(\Delta(m)<0)$, there are exactly 2 normal vectors $b_{1}, b_{2}$ at $f(m)$ such that $m$ is a degenerate critical point of their corresponding height functions.

c) At a parabolic point $(\Delta(m)=0)$ there is a unique normal vector $b$ such that $f_{b}$ is degenerate at $m$.

d) A parabolic point $m$ is an umbilic point for $f_{b} \Leftrightarrow m$ is an inflection point of $M$.

In the cases b), c) and d) above, the normal directions $b$ for which $f_{b}$ has a degenerate singularity were called binormals of $M$. We also saw in [13] that given such a binormal at a point $f(m)$, the point $f(m)+\epsilon b$ on the canal hypersurface $C M$ of $f(M)$ is parabolic and the projection of the principal direction of vanishing curvature at this point, under the differential of the natural projection map $\xi$ : $C M \rightarrow M$, is precisely the asymptotic direction of $f(M)$ associated to the binormal direction $b$ at $f(m)$. Now, if in the case b) we denote by $\gamma$ the normal section of $M$ tangent to the asymptotic direction $\theta$ at $f(m)$ associated to one of the binormal vectors $b_{i}$. Then $m$ is a fold singularity of $f_{b_{i}}$ if and only if $\gamma$ has nonvanishing normal torsion at $\mathrm{m}$. A parabolic point, which is not an inflection point of $M$, is either a fold or a cusp singularity of $f_{v}$ and

i) $m$ is a fold singularity of $f_{v} \Leftrightarrow \theta$ is transversal to the curve $\Delta^{-1}(0)$ of parabolic points of $f(M)$.

ii) $m$ is a cusp singularity of $f_{v} \Leftrightarrow \theta$ is tangent to $\Delta^{-1}(0)$. (These points will be called parabolic cusps of $M$ ).

When $m$ is an inflection point,

i) $m$ is a normal crossing point of $\Delta^{-1}(0) \Leftrightarrow m$ is an inflection point of real type;

ii) $m$ is an isolated point of $\Delta^{-1}(0) \Leftrightarrow m$ is an inflection point of imaginary type.

As a consequence of this local analysis we obtained the following global results for generic surfaces in 4 -space:

1) The set of hyperbolic points is a nonempty open region of $M$.

2) If $f(M)$ has no inflection points, then the curve $\Delta^{-1}(0)$ is a disjoint union of circles.

3) The inflection points and the parabolic cusps are isolated on $M$.

4) If $f(M)$ is locally convex (i.e., has a locally supported hyperplane at each point), then $M$ has no elliptic points (i.e., $\Delta \leq 0)$ and $\Delta^{-1}(0)$ consist only of isolated points of $M$ (i.e., $M$ can only have inflection points of imaginary type).

5) Any locally convex embedded 2-sphere has inflection points. 
In the next sections we analyze the behaviour of the fields of asymptotic directions in order to obtain some relations between the number of inflection points of the surface and its topology.

\section{Asymptotic Lines ON SURfaCes in $\mathbb{R}^{4}$}

From the results described in the previous section, we can conclude that a generically embedded surface $f(M)$ always has an open region, made of hyperbolic points, at which there are defined two line fields, $V_{1}$ and $V_{2}$, of asymptotic directions. These two fields "collapse" onto a unique one over the curve $\Delta^{-1}(0)$.

Their integral lines are called asymptotic lines and their singular points coincide with the inflection points of $M$.

Lemma 3.1. Let $f: \mathbb{R}^{2}, 0 \rightarrow \mathbb{R}^{4}, 0$ be the local parametrization of $M$ given by $\left(x, y, f_{1}(x, y), f_{2}(x, y)\right)$. Then the differential equation of the asymptotic lines of $M$ has the form

$$
A(x, y)(\dot{x})^{2}+B(x, y)(\dot{x})(\dot{y})+C(x, y)(\dot{y})^{2}=0
$$

where

$$
\begin{aligned}
& A(x, y)=f_{1_{x y}} f_{2_{x x}}-f_{1_{x x}} f_{2_{x y}}, \\
& B(x, y)=f_{1_{y y}} f_{2_{x x}}-f_{1_{x x}} f_{2_{y y}}, \text { and } \\
& C(x, y)=f_{1_{y y}} f_{2_{x y}}-f_{1_{x y}} f_{2_{y y}} .
\end{aligned}
$$

Proof. Let

$$
\begin{aligned}
\mathbb{R}, 0 & \stackrel{\alpha}{\rightarrow} \mathbb{R}^{2}, 0 \\
s & \mapsto(x(s), y(s))
\end{aligned}
$$

be a local parametrization by arclength of an asymptotic curve of $M$ in a neighbourhood of $p$. Its image in $f(M)$ is given by

$$
\begin{aligned}
\mathbb{R}, 0 & \stackrel{\gamma=f \circ \alpha}{\longrightarrow} \mathbb{R}^{4}, 0 \\
(s) & \mapsto\left(x(s), y(s), f_{1}(x(s), y(s)), f_{2}(x(s), y(s))\right) .
\end{aligned}
$$

To obtain the parametrization of the curvature ellipse along $\gamma(s)$, we choose the orthonormal frame $\left\{e_{1}, e_{2}, e_{3}, e_{4}\right\}, e_{i}=\frac{g_{i}}{\left|g_{i}\right|}$, where $g_{1}=\left(1,0, f_{1_{x}}, f_{2_{x}}\right), g_{2}=$ $\left(0,1, f_{1 y}, f_{2 y}\right), g_{3}=\left(-f_{1 x},-f_{1 y}, 1,0\right)$ and $g_{4}=\left(-f_{2_{x}}-f_{1_{y}}\left(f_{2 x} f_{1 y}-f_{1_{x}} f_{2 y}\right),-f_{2 y}-\right.$ $\left.f_{1_{x}}\left(f_{1_{x}} f_{2_{y}}-f_{1_{y}} f_{2_{x}}\right),-f_{1_{x}} f_{2_{x}}-f_{1_{y}} f_{2 y}, 1+{f_{1 x}}^{2}+f_{2_{x}}{ }^{2}\right)$.

With respect to this frame we obtain

$$
\begin{aligned}
& \eta(s, \theta)=\left(f_{1_{x x}} \cos ^{2} \theta+2 f_{1_{x y}} \cos \theta \sin \theta+f_{1_{y y}} \sin ^{2} \theta\right) e_{3} \\
& +\left(R \cos ^{2} \theta+2 S \cos \theta \sin \theta+T \sin ^{2} \theta\right) e_{4}
\end{aligned}
$$

where

$$
\begin{aligned}
& R=f_{2_{x x}}\left(1+f_{1_{x}}{ }^{2}+f_{1_{y}}{ }^{2}\right)-f_{1_{x x}}\left(f_{1_{x}} f_{2_{x}}+f_{1_{y}} f_{2_{y}}\right), \\
& S=f_{2_{x y}}\left(1+{f_{1_{x}}}^{2}+{f_{1_{y}}}^{2}\right)-f_{1_{x y}}\left(f_{1_{x}} f_{2_{x}}+f_{1_{y}} f_{2_{y}}\right), \\
& T=f_{2_{y y}}\left(1+{f_{1_{x}}}^{2}+{f_{1 y}}^{2}\right)-f_{1_{y y}}\left(f_{1_{x}} f_{2_{x}}+f_{1_{y}} f_{2_{y}}\right) .
\end{aligned}
$$

The asymptotic direction $\theta=\theta(s)$ at $\gamma(s)$ is the solution of the equation

$$
\eta(s, \theta)=\lambda \frac{\partial \eta}{\partial \theta}(s, \theta) .
$$

The result now follows by a straightforward calculation and elimination of $\lambda$. 

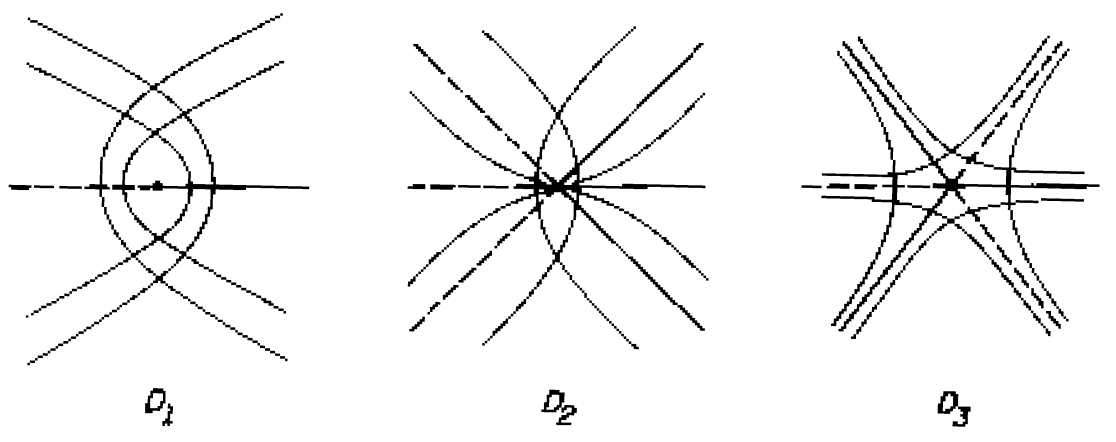

Figure 1. Asymptotic lines in the neighbourhood of an inflection point of imaginary type

The binary differential equation $A(x, y)(\dot{x})^{2}+B(x, y)(\dot{x})(\dot{y})+C(x, y)(\dot{y})^{2}=0$, has been considered by many authors. The local behavior of this equation in the case that 0 is a regular value of the discriminant $B^{2}-4 A C$, was studied by $\mathrm{T}$. Banchoff and R. Thom [1, L. Dara [5] and A. Davydov [7. At points of Morse type of $B^{2}-4 A C$, the generic behavior was obtained by V. Guiñez [9], and J. Bruce and F. Tari 2]. The special case of the differential equation of lines of curvature was first considered G. Darboux [6], and recently by C. Gutierrez and J. Sotomayor [10], and J. Bruce and D. Fidal [4].

In the following lemma, we discuss the normal forms of the differential equation of asymptotic lines in the neighbourhood of an inflection point of imaginary type.

Lemma 3.2. For a generic embedding, the differential equation of asymptotic lines has the following normal form in the neighbourhood of an inflection point of imaginary type:

$$
\begin{aligned}
\left(b x-a P_{3} y+\ldots\right)(\dot{x})^{2} & +\left[\left(c-a P_{3}\right) x+\ldots\right](\dot{x})(\dot{y}) \\
& +\left[-b P_{3} x+c P_{3} y+\ldots\right](\dot{y})^{2}=0,
\end{aligned}
$$

where $a=\frac{1}{2} f_{1_{x x}}(0,0), b=\frac{1}{2} f_{1 x y}(0,0), c=\frac{1}{2} f_{1 y y}(0,0)$ and $P_{3}=\frac{1}{6} f_{2 x x x}(0,0)$.

The singular point $(0,0)$ is a Darbouxian umbilic of type $D_{1}, D_{2}, D_{3}$, as in Figure 1. Moreover, the index of the singular point is $1 / 2$ in case $D_{1}$ and $D_{2}$, and $-1 / 2$ in case $D_{3}$.

Proof. At an inflection point of imaginary type, $A(0,0)=B(0,0)=C(0,0)=0$, and we will show that the discriminant of the differential equation (3.2) has a nondegenerate minimum. The local classification of binary equations under these hypotheses is well known (see 2, Theorem 4.1, or [10). The singular point $(0,0)$ is a Darbouxian umbilic of type $D_{1}, D_{2}$ or $D_{3}$ (Figure 1).

To prove this, we first compute the discriminant $B^{2}-4 A C$ to find that

$$
\left(B^{2}-4 A C\right)(x, y)=-4 \Delta(x, y) .
$$

Now, we write $f$ in the neighbourhood of $(0,0)$ as $\left(x, y, f_{1}, f_{2}\right)$, where

$$
f_{1}(x, y)=a x^{2}+2 b x y+c y^{2}+M_{1} x^{3}+3 M_{2} x^{2} y+3 M_{3} x y^{2}+M_{4} y^{3}+\epsilon_{1}(x, y)
$$

and

$$
f_{2}(x, y)=e x^{2}+2 f x y+g y^{2}+P_{1} x^{3}+3 P_{2} x^{2} y+3 P_{3} x y^{2}+P_{4} y^{3}+\epsilon_{2}(x, y)
$$

with $\epsilon_{i} \in m^{4}, i=1,2$. 
Since $(0,0)$ is an inflection point, there exists a binormal direction $b$ such that the height function in this direction has a singularity of umbilic type. Choosing $b=e_{4}=(0,0,0,1)$, by a convenient choice of chart, we can assume that $f_{2}(x, y)=$ $x^{3}+3 P_{3} x y^{2}$, with $P_{3} \neq 0$.

Thus, the differential equation of asymptotic lines becomes

$$
\left[b x-a P_{3} y+\ldots\right](\dot{x})^{2}+\left[\left(c-a P_{3}\right) x+\ldots\right](\dot{x})(\dot{y})+\left[-b P_{3} x+c P_{3} y+\ldots\right](\dot{y})^{2}=0 \text {. }
$$

The discriminant of this equation is

$$
\left[\left(c-a P_{3}\right) x+\ldots\right]^{2}-4\left[b x-a P_{3} y+\ldots\right]\left[-b P_{3} x+c P_{3} y+\ldots\right]
$$

and computing its Hessian at $(0,0)$ we find

$$
16 k(0,0)\left(c-a P_{3}\right)^{2} P_{3}{ }^{2} .
$$

Since the Gaussian curvature $k(x, y)$ is positive at an umbilic point of imaginary type, it follows that the discriminant has a nondegenerate minimum at $(0,0)$.

To compute the index, we use Bendixson's formula,

$$
I=1+\frac{e-h}{2}
$$

where $e$ and $h$ represent, respectively, the number of elliptic and hyperbolic sectors arriving at the singular point. Now the assertion follows immediately by observing that in cases $D_{1}$ and $D_{3}$ these sectors are as shown in Figure 1 and all of them are hyperbolic; in case $D_{2}$, one is hyperbolic and the other one is parabolic.

From the results described in the first section we deduce that the asymptotic lines are transversal to the smooth part of the curve $\Delta^{-1}(0)$ except at the parabolic cusps of $M$, where they are tangent. Now, if we assume that there are no inflection points of real type, the closure of the set $M_{h}$ of hyperbolic points will be a regular compact surface whose boundary is the embedded curve $\Delta^{-1}(0)=\{$ inflection points of imaginary type $\}$ (unless $f(M)$ is convex, in which case $M=\bar{M}_{h}$ ). Thus, we can view any of the two fields of asymptotic directions as a line field defined on a compact manifold (with boundary or not depending on the convexity properties of the surface). At the boundary points, these two directions coincide and the resulting direction is transversal to the curve $\Delta^{-1}(0)$, except possibly on a finite number of parabolic cusp points.

\section{INFLECTION POINTS AND EULER NUMBERS}

In this section we use a generalization of the Poincare formula for line fields on surfaces in order to obtain certain conclusions about the numbers of inflection points and of parabolic cusps. In Theorem 4.1 we obtain an extension of an earlier result of E.A. Feldman [8] on the number of umbilics on a generic compact surface in 3-space. An interesting Feldman-type result for surfaces in $\mathbb{R}^{3}$ is obtained by B. Smith and F. Xavier [19].

Given a smooth line field $V$ on a surface $S$, assume that $V$ has finitely many critical points all contained in the interior of $S$, and finitely many inner and outer contact points with the boundary of $S$ (see [1] for definitions). Then the sum, $\operatorname{Ind}(V)$, of the indices at the critical points of $V$ is given by the formula

$$
\operatorname{Ind}(V)=\chi(S)+\frac{\sigma-\nu}{2}
$$


where $\sigma$ and $\nu$ are the numbers of inner and outer contact points, and $\chi(S)$ denotes the Euler number of $S$ (see [1], [12]). We observe that this formula is valid for both orientable and nonorientable surfaces. Moreover, if $S$ has no boundary, we have $\sigma=\nu=0$ and the expression reduces to the well known Poincaré-Hopf formula.

Theorem 4.1. If $f: M \rightarrow \mathbb{R}^{4}$ is a generic locally convex embedding of a (nonnecessarily orientable) closed surface $M$ in $\mathbb{R}^{4}$, then

$$
2|\chi(M)| \leq \sharp\{\text { inflection points }\}
$$

Proof. Let $V$ be one of the line fields of asymptotic directions on $M$. Since $f(M)$ is locally convex, we have that $V$ is defined on the whole of $M$. Moreover, the singularities of $V$ are the inflection points of $M$, which can only be isolated points, $p_{1}, p_{2}, \ldots, p_{n}$, of the set $\Delta^{-1}(0)$ (inflections of imaginary type). Now the above formula in the case of surfaces without boundary leads to

$$
|\chi(M)| \leq \sum_{i=1}^{n}\left|\operatorname{ind} V\left(p_{i}\right)\right|
$$

but $\mid$ ind $V\left(p_{i}\right) \mid=\frac{1}{2}, \forall i=1, \ldots, n$, so we get

$$
|\chi(M)| \leq \frac{\sharp \text { inflection points }\}}{2} \text {. }
$$

Since any generic locally convex surface in 4-space is orientable (see Remark 2.3 in [18), our main result follows now as an immediate consequence of the above theorem.

Corollary 4.1. Any generic embedding of a compact locally convex surface, with Euler number $\chi(M) \neq 0$ has at least 4 inflection points.

Remark 4.1. Notice that both Theorem4.1 and Corollary 4.1 still hold for a generic immersed surface $f(M)$, with transverse self-intersections. In fact, we can pull back the asymptotic directions of $f(M)$ to $M$ by the immersion $f$. Observe that, generically, the self-intersections of $f(M)$ will not coincide with the inflection points and then we have two well defined fields of directions on $M$, whose singularities are mapped bijectively by $f$ onto the inflection points of $f(M)$. We must point out that, in this case, we may not have $\chi(M)=\chi(f(M))$.

When the surface $f(M)$ is nonlocally convex, the region of elliptic points is nonempty, and hence the line fields of asymptotic directions are not defined on the whole $M$, but on the compact surface with boundary obtained by taking the closure of the hyperbolic region of $M$. If there are no inflection points of real type, $\bar{M}_{h}$ is a regular surface and we can apply the generalized Poincaré-Hopf formula, getting the following:

Theorem 4.2. If $f: M \rightarrow \mathbb{R}^{4}$ is a generic embedding of a (nonnecessarily orientable) closed surface $M$ in $\mathbb{R}^{4}$ with no inflection points of real type, then

$$
2\left|\chi\left(\bar{M}_{h}\right)\right| \leq \sharp\{\text { inflection points }\}+\sharp\{\text { parabolic cusps }\} .
$$

Then we can assert:

Corollary 4.2. If $\chi\left(\bar{M}_{h}\right) \neq 0$, then $M$ has either parabolic cusps or inflection points. 
Lemma 4.1. Let $n_{r}$ be the number of inflection points of real type of a generic embedding of a closed surface $M$ in $\mathbb{R}^{4}$. Then

$$
\chi\left(M_{e}\right)+\chi\left(M_{h}^{*}\right)=\chi(M)+n_{r}
$$

where $M_{h}^{*}$ represents the union of the set $M_{h}$ of hyperbolic points with the inflection points of $M$ of imaginary type.

Proof. The sets $M_{e}$ (elliptic points), $M_{h}^{*}, M_{0}$ (noninflection parabolic points) and $U_{r}$ (umbilic points of real type) determine a stratification of the surface $M$. The connected components of the subsets $M_{e}$ and $M_{h}^{*}$ being the 2-dimensional strata, those of $M_{0}$, being the 1-dimensional strata and $U_{r}$ the 0 -dimensional ones. We can now apply a standard formula relating the Euler numbers of the strata with that of $M$ [17], obtaining that

$$
\chi\left(M_{e}\right)+\chi\left(M_{h}^{*}\right)-\chi\left(M_{0}\right)+\chi\left(U_{r}\right)=\chi(M) .
$$

But $\chi\left(M_{0}\right)$ is given by the number of simply connected curves in $M_{0}$. On the other hand, there are 2 of these curves for each inflection point of real type. Hence $\chi\left(M_{0}\right)=2 n_{r}=2 \chi\left(U_{r}\right)$ from which the result follows.

The following corollary follows from this lemma and Theorem 4.2 .

Corollary 4.3. If we represent by $\operatorname{Reg}\left(M_{h}\right)$ the union of connected components of $M_{h}$ whose closures are regular compact surfaces (so they do not contain inflection points of real type of $M$ ), then

$$
N \geq 2\left|\chi\left(\overline{\operatorname{Reg}\left(M_{h}\right)}\right)\right|+\chi\left(M_{e}\right)+\chi\left(M_{h}^{*}\right)-\chi(M),
$$

where $N$ denotes the sum of the numbers of inflection points of both real and imaginary type and parabolic cusps of $M$.

Let $\mathcal{K}_{c}: C M \rightarrow \mathbb{R}$ be the Gaussian curvature function on the canal 3-manifold, $C M$, of $M$. The parabolic set, $\mathcal{K}_{c}^{-1}(0)$, of $C M$ is the singular set of the Gauss map $\Gamma: C M \rightarrow S^{3}$. It can be shown [13] that for a generic embedding of a surface $M$, the set $\mathcal{K}_{c}^{-1}(0)$ is a regular surface except by a finite number of points $(m, v)$ which are singularities of type $\sum^{2,0}$ of $\Gamma$, or equivalently, umbilic points $\left(D_{4}^{ \pm}\right)$of the height function $\tilde{f}_{v}$ (i.e., $m$ is an inflection point of $M$ ).

Let $\xi: C M \rightarrow M$ be the natural projection of $C M$ onto $M$, i.e., $\xi(m, v)=m$. The image of the set of parabolic points $\mathcal{K}_{c}^{-1}(0)$ by $\xi$ is the set $\Delta \leq 0$. If we denote by $\bar{\xi}$ the restriction of $\xi$ to the surface $\mathcal{K}_{c}^{-1}(0)-\sum^{2}(\Gamma)$, and consider $B=\{(m, v) \in$ $\left.\mathcal{K}_{c}^{-1}(0): m \in M_{h}\right\}$, we have

(i) $\bar{\xi}_{\mid B}: B \rightarrow M_{h}$ is a local diffeomorphism, more precisely, it is a double covering.

(ii) $\Delta(m)=0$ and $m$ is not an inflection point $\Leftrightarrow$ there is $v \in S^{3}$ such that $(m, v)$ is a singular point (fold) of $\bar{\xi}$.

(See [13] for a proof.)

Theorem 4.3. Suppose that $f: M \rightarrow \mathbb{R}^{4}$ is a generic embedding of a closed surface $M$ in $\mathbb{R}^{4}$ for which $N=0$, then

a) If $M$ is orientable, the parabolic subset $\mathcal{K}_{c}^{-1}(0)$ of the canal 3-manifold, $C M$, of $M$ in $\mathbb{R}^{4}$ is homeomorphic to $n$ copies of a torus. With $n=2$ when $M$ is locally convex.

b) If $M$ is nonorientable, $\mathcal{K}_{c}^{-1}(0)$ is homeomorphic to:

b1) $n$ copies of the Klein bottle $(n>0)$, or

b2) $n$ copies of the Klein bottle and $m$ copies of the torus $(n>0, m \geq 0)$. 
Proof. It follows from Theorem 4.2 that $\chi\left(\bar{M}_{h}\right)=0$. On the other hand, $\operatorname{Reg}\left(M_{h}\right)$ $=M_{h}$, for there are no inflection points of real type. Hence the closure of $M_{h}$ is a compact surface with or without boundary according to whether or not $M$ is locally convex.

The classification theorem of compact surfaces tells us that:

a) If $M_{h}$ is orientable and connected, then it must be homeomorphic to the surface $T_{g, b}$, of genus $g$ obtained as the connected sum of $g$ tori (or a 2-sphere if $g=0)$ minus a finite number $b>0$ of open discs, with $\chi\left(\bar{M}_{h}\right)=2-2 g-b=0$.

b) If $M_{h}$ is connected and nonorientable, then it must be homeomorphic to the surface $P_{g, b}$, of genus $g$ obtained as the connected sum of $g$ real projective planes $(g>1)$ minus a finite number $b>0$ of open discs, with $2-g-b=0$.

From this we get the following possibilities:

In case a): $b=0$ and $g=1$ and in this case $M=M_{h}$, and $f$ is a locally convex embedding of the torus, or $b=2$ and $g=0$ and $M_{h}$ is a cylinder.

In case b) we could have either $b=0$ and $g=2$, or $b=g=1$. The first case cannot occur for we would have $M_{h}=M$ and this contradicts the fact that a locally convex surface is always orientable. In the second case, $M_{h}$ is a Möbius strip.

Now, since the regular points of the projection $\bar{\xi}: \mathcal{K}_{c}^{-1}(0) \rightarrow M_{h}$ form a double covering of $M_{h}-M_{0}$ with the 2 sheets glued along their boundaries (when these exist), the result follows from observing that we can apply the above argument to each connected component of $M_{h}$, and that gluing 2 cylinders or Möbius strips in this way gives a torus or a Klein bottle, respectively.

Remark 4.2. While it is easy to find examples of a torus embedded in 4-space with $N=0$, we have not been able to find a single example of a Klein bottle with this property.

Remark 4.3. The singular set of the Gauss map $\Gamma$ coincides with the set of degenerate singularities of the family of height functions on $M$; the image through $\Gamma$ of this set is known as the bifurcation set of this family. Under the hypothesis of the Theorem 4.3, we deduce that this set will be a topologically immersed collection of tori or Klein bottles (with corners corresponding to cusps of height functions) .

Remark 4.4. If the embedding $f: M \rightarrow \mathbb{R}^{4}$ is such that the most degenerate singularities of its associated height functions are folds, we have trivially that $N=0$ and thus all of the above conclusions apply. Moreover, since there are no cusps of height functions in this case, the above mentioned tori or Klein bottles will be immersed (without corners).

Remark 4.5. When $M$ is locally convex, the set $\mathcal{K}_{c}^{-1}(0)$ has exactly 2 connected components (given by the 2 leaves of the double covering map $\bar{\xi}: \mathcal{K}_{c}^{-1}(0) \rightarrow M$ ). Observe that this is not a sufficient condition for local convexity.

\section{EXAMPLES}

We discuss below three classes of examples of orientable surfaces in 4-space: convex surfaces, complex curves and $S^{1}$-bundles.

5.1. Convex surfaces. Examples of convex surfaces in 4-space are obtained as images of surfaces in 3-space through the inverse of stereographic projection $\pi$ : $\mathbb{R}^{3} \rightarrow \mathbb{R}^{4}$. In fact, the singular points (umbilics) of the principal direction fields of any surface $M$ in 3 -space are taken by $\pi$ to singular points (inflection points) 

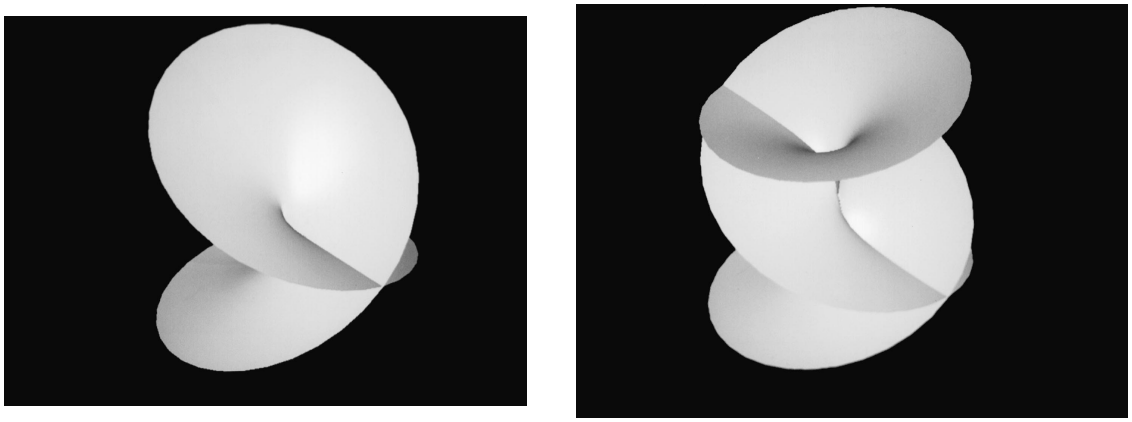

Figure 2. Projections in 3-space of $z+w^{3}=0$
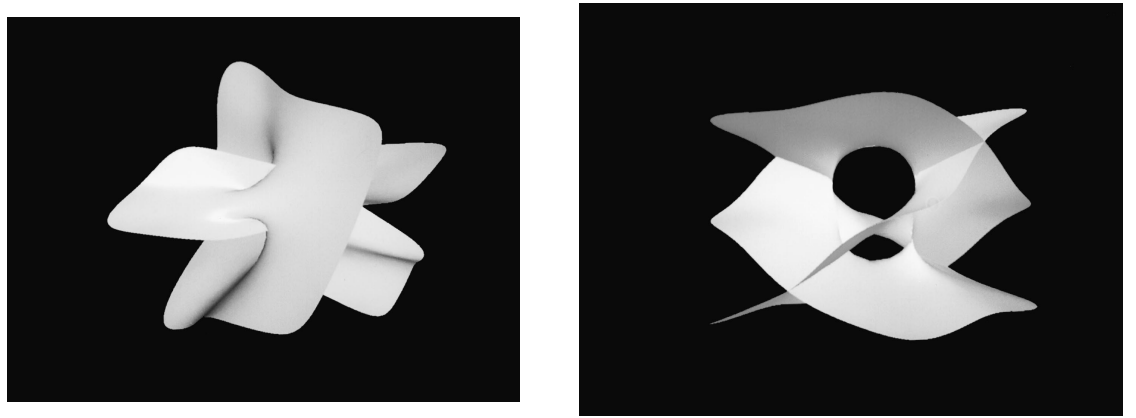

FiguRE 3. Projections in 3-space of $z+w^{3}=0$

of the asymptotic direction fields on $\pi(M)$, considered as a surface in 4-space. In particular, any ellipsoid with three distinct axes is taken by $\pi$ to a convexly embedded 2 -sphere in $\mathbb{R}^{4}$ with 4 inflection points. On the other hand, the image through $\pi$ of the standard torus in $\mathbb{R}^{3}$ provides a (nongeneric !) example of a closed convex surface with vanishing Euler characteristic and no inflection points in $\mathbb{R}^{4}$.

5.2. Complex curves. An interesting class of examples of surfaces with boundary in 4-space is given by an algebraic regular complex curve in $\mathbb{C}^{2}$, defined as a level set of a polynomial function $f: \mathbb{C}^{2} \rightarrow \mathbb{C}$.

Figures 2 and 3 represent projections in 3 -space of a level curve of the functions $f(z, w)=z+w^{3}$ and $f(z, w)=z^{2}+w^{3}$, respectively. These are surfaces completely made of elliptic points $(\Delta>0)$.

Another kind of example is obtained by considering $S^{1}$-bundles over closed curves in 4-space, the fibres lying in the normal space of the curve at each point. These are tori that may be convex or not, depending on the choice of the circles in the normal bundle of the curve.

A nonconvex example is provided by the following torus:

$$
(x, y) \rightarrow\left(f_{1}(x, y), f_{2}(x, y), f_{3}(x, y), f_{4}(x, y)\right),
$$

where

$$
\begin{aligned}
& f_{1}(x, y)=(1-1 / 10 \cos (y)) \cos (x)+1 / 10 \sin (x) \sin (y), \\
& f_{2}(x, y)=(1-1 / 10 \cos (y)) \sin (x)-1 / 10 \cos (x) \sin (y),
\end{aligned}
$$



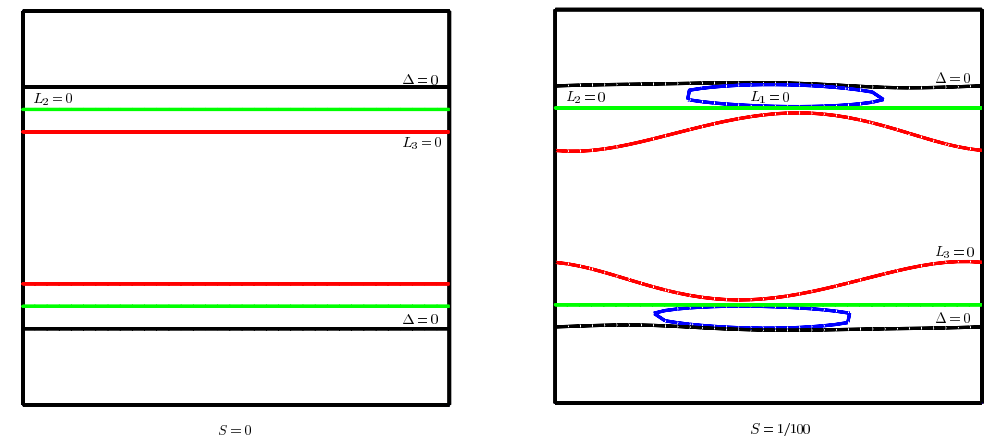

Figure 4. Parabolic curve $\Delta=0$, and the zero sets of $L_{1}, L_{2}, L_{3}$.

$$
\begin{aligned}
& f_{3}(x, y)=(1-2 / 5 \cos (y)) \cos (2 x)+4 / 5 \sin (2 x) \sin (y), \\
& f_{4}(x, y)=(1-2 / 5 \cos (y)) \sin (2 x)-4 / 5 \cos (2 x) \sin (y) .
\end{aligned}
$$

This has a parabolic set made of two nonhomotopically trivial closed curves, and does not present any inflection points; neither are parabolic points. So, in this case, $M_{h}$ is homeomorphic to an annulus and $N=0$.

Let $f_{s}$ be the one-parameter perturbation of $f$, given by

$$
(x, y) \rightarrow\left(f_{1}(x, y, s), f_{2}(x, y), f_{3}(x, y), f_{4}(x, y)\right),
$$

where

$$
\begin{gathered}
f_{1}(x, y, s)=(1-1 / 10 \cos (y)) \cos (x)+1 / 10 \sin (x) \sin (y)+s \cos (y), \\
f_{2}(x, y)=(1-1 / 10 \cos (y)) \sin (x)-1 / 10 \cos (x) \sin (y), \\
f_{3}(x, y)=(1-2 / 5 \cos (y)) \cos (2 x)+4 / 5 \sin (2 x) \sin (y), \\
f_{4}(x, y)=(1-2 / 5 \cos (y)) \sin (2 x)-4 / 5 \cos (2 x) \sin (y) .
\end{gathered}
$$

The inflection points occur at points where the second fundamental form drops rank, hence they correspond to the common solutions of the $2 \times 2$ minors of the matrix

$$
\alpha(m)=\left(\begin{array}{lll}
a & b & c \\
e & f & g
\end{array}\right)(m)
$$

that is, to the solutions of

$$
a f-b e=0, a g-c e=0, b g-c f=0 .
$$

With the support of the softwares MAPLE and LSMP (Liverpool Surface Modeling Package)[15], we present a numerical study of the singularities of the above family for several values of the parameter $s$.

When $0 \leq s \leq 1 / 75, f_{s}$ has no inflection points or parabolic cusps, hence $N=0$ and $M_{h}$ is homeomorphic to an annulus. Figure 4 shows the curves $\Delta=0, L_{1}=$ $0, L_{2}=0$ and $L_{3}=0$, where $L_{i}$ correspond, respectively, to $a f-b e, b g-c f$ and $a g-c e$, for $s=0$ and $s=1 / 100$.

There exist two symmetric bifurcation points for some value of $s \in(1 / 100,1 / 75)$. These bifurcation points are generic and correspond to the creation of two symmetric isolated Morse points of the function $\Delta$. 

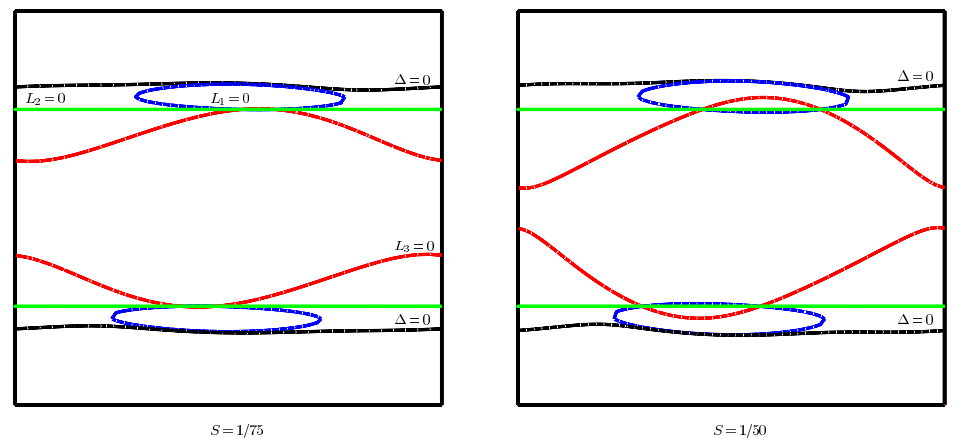

Figure 5. Examples of embeddings of tori presenting 4 inflection points $P_{1}, P_{2}, P_{3}, P_{4}$, of imaginary type.
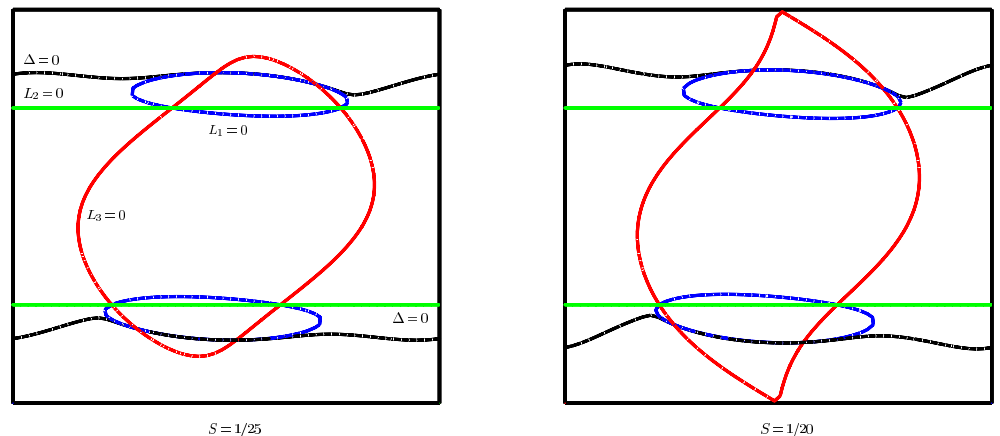

FiguRE 6. Examples of embeddings of tori presenting 4 inflection points $P_{1}, P_{2}, P_{3}, P_{4}$, of imaginary type.

When $s=1 / 50, f_{s}$ has 4 inflection points of imaginary type, $P_{1}, P_{2}, P_{3}, P_{4}$, where

$$
\begin{gathered}
P_{1}=\left(2 \arctan (-5 / 3-1 / 3 \sqrt{79}, \pi / 2) P_{2}=(2 \arctan (-5 / 3+1 / 3 \sqrt{79}, \pi / 2),\right. \\
P_{3}=\left(2 \arctan (-5 / 3-1 / 3 \sqrt{79}, 3 \pi / 2) P_{4}=(2 \arctan (-5 / 3+1 / 3 \sqrt{79}, 3 \pi / 2) .\right.
\end{gathered}
$$

To compute their indices, we use Proposition 2.4 in [16], where a characterization of the Darbouxian singularities in terms of the 1-jet of the coefficients $A, B, C$, of the equation $A(x, y) d x^{2}+2 B(x, y) d x d y+C(x, y) d y^{2}=0$, is given. We obtain that index $\left(P_{1}\right)=\operatorname{index}\left(P_{3}\right)=-1 / 2$, and $\operatorname{index}\left(P_{2}\right)=\operatorname{index}\left(P_{4}\right)=1 / 2$.

This stable behaviour seems to hold for all values of $s, 1 / 75 \leq s \leq 1 / 20$. Notice that in this case $M_{h}$ is still an annulus, and we can verify that the sum, $\operatorname{Ind}(V)$, of the indices at the inflection points is the Euler characteristic of $M_{h}$ (Figures 5 ) and 6).

The next symmetric bifurcations hold for some value of $s$ close to $1 / 12$. These seem to be generic bifurcations of cusp type (see [3]). For $1 / 9 \leq s \leq 1 / 5$, the surface has 4 inflection points $P_{1}, P_{2}, P_{3}, P_{4} ; P_{1}$ and $P_{3}$ are inflection points of real type, $P_{2}$ and $P_{4}$ are of imaginary type (Figures 7 and 8 ). Our results relating the topology of the surface and the singularities of the asymptotic fields do not apply to this case.

We also analyzed the behavior of the family for values of $s, 1 / 5 \leq s \leq 1$. However, it seems that the family is not generic in this interval. Indeed, in Figure 9 we see 


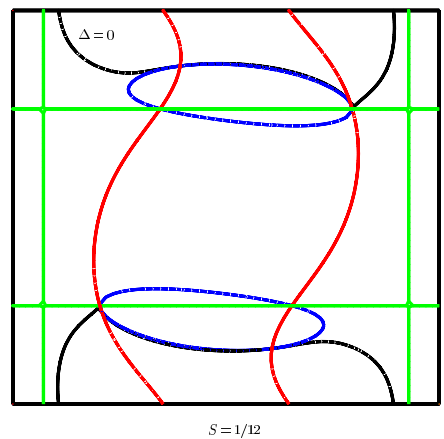

Figure 7. Bifurcation of cusp type

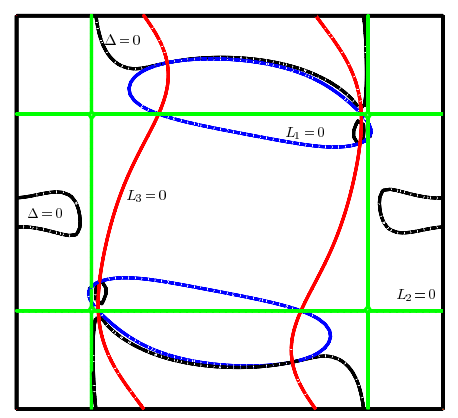

$S=1 / 6$

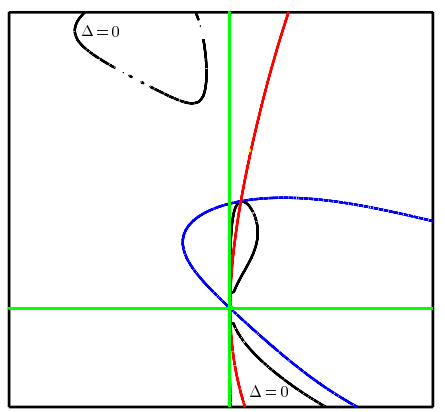

$S=1 / 5$

FiguRE 8 . Example presenting 4 inflection points $P_{1}, P_{3}$, of real type, $P_{2}, P_{4}$, of imaginary type.

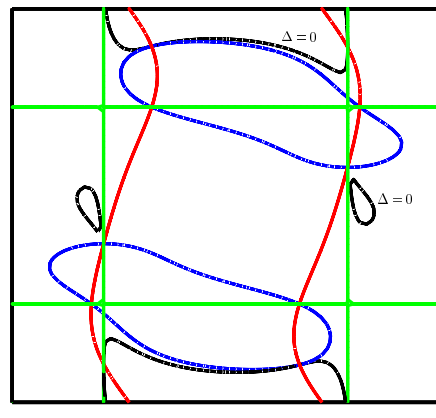

$S=1 / 3$

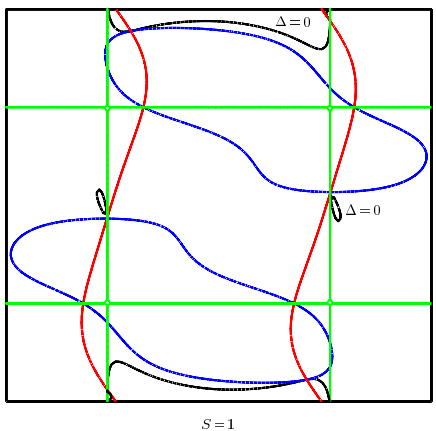

$S=1$

Figure 9. Creation of two degenerate inflection points

that for $s=1 / 3$ and $s=1$, the curves $L_{1}, L_{2}$ and $L_{3}$, seem to intersect in two more symmetric points. But, Figures $10 \mathrm{k}$ and $10 \mathrm{~b}$ show that the curve $k=0$ may intersect them, which indicates that these new inflection points are of flat type. We shall discuss these cases in a later paper.

The examples of nonorientable closed surfaces in 4-space that were analyzed turned out to be nongeneric, all of them having nonisolated inflection points. 


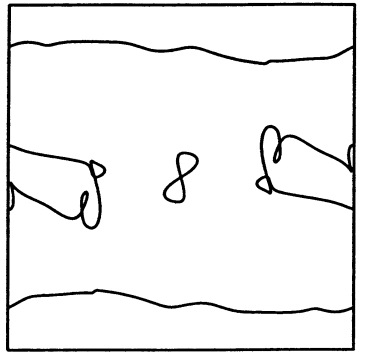

(a) The curve $k=0$

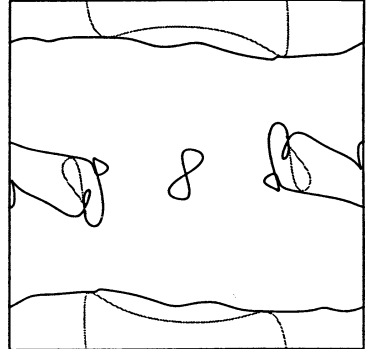

(b) Intersection of the curves $\Delta=0$
and $k=0$

Figure 10.

This seems to be due to the symmetry of their parametrizations. Some attempts at perturbing their symmetry, in order to make them generic, failed because the computations (using MAPLE software) became unmanageable.

\section{ACKNOWLEDGMent}

We are very much indebted to Dr. Richard Morris, whose computer package 'Liverpool Surface Modeling Package' produced the configurations of the parabolic curve and umbilic points reproduced in the paper. We also thank Professor Antonio Castelo Filho for kindly lending us the pictures of the projections of the complex curves. These were produced with his package 'Medusa'.

\section{REFERENCES}

1. T. Banchoff and R. Thom, Erratum et compléments: Sur les points paraboliques des surfaces, C. R. Acad. Sci. Paris Sér. A-B, 291 (1980), 503A-505B. MR 82a:53003b

2. J. W. Bruce and F. Tari, On binary differential equations, Nonlinearity, 8 (1995), 255-271. MR 96d:58124

3. J. W. Bruce and F. Tari, Generic 1-parameter families of binary differential equations, Discrete and Continuous Dynamical Systems, 3, no.1 (1997), 79-90. MR 98h:58123

4. J. W. Bruce and D. Fidal, On binary differential equations and umbilics, Proc. Royal Soc. Edinburgh, Sect. A 11 (1989), 147-168. MR 90e:58141

5. L. Dara, Singularités génériques des equations differentielles multiformes, Bol. Soc. Brasil. Mat. 6 (1975), 95-128. MR 58:7720

6. G. Darboux, Sur la forme des lignes de courbure dans le voisinage d'un ombilic, Leçons de sur la Théorie des Surfaces, IV, Note 7, Gauthiers-Villars, Paris, 1896.

7. A. A. Davydov, Normal forms of differential equation that is not solved with respect to the derivative in a neighbourhood of its singular point, Funct. Anal. Appl. 19 (1985), 1-10. MR 87d:58116

8. E. A. Feldman, On parabolic and umbilic points of immersed hypersurfaces, Trans. Amer. Math. Soc. 127 (1967), 1-28. MR 34:6790

9. V. Guiñez, Positive quadratic differential forms and foliations with singularities on surfaces. Trans. Amer. Math. Soc. 309, no. 2 (1988), 477-502. MR 89h:57021

10. C. Gutierrez and J. Sotomayor, Structurally stable configurations of lines of principal curvature, Astèrisque, 98-99 (1982). MR 85h:58006

11. J. A. Little, On singularities of submanifolds of higher dimensional Euclidean space. Ann. Mat. Pura Appl. (ser. 4A) 83 (1969), 261-335. MR 42:6851

12. J. Llibre and Y. Yanquian, On the dynamics of surface vector fields and homeomorphisms. Bulletin de la Societé Mathématique de Belgique (to appear). 
13. D. K. H. Mochida, M. C. Romero Fuster, and M. A. Ruas, The geometry of surfaces in 4-space from a contact viewpoint. Geometriae Dedicata 54 (1995), 323-332. MR 96d:58013

14. D. Mond, Thesis, Liverpool (1982).

15. R. J. Morris, The use of computer graphics for solving problems in singularity theory. Visualization and Mathematics, Edited by H. Hege and K. Polthie, Springer-Verlag, Heidelberg, 1997, 53-66. CMP 98:10

16. A. C. Nabarro, Equações Diferenciais Binárias e Geometria Diferencial, M.Sc. thesis, ICMSC-USP, (1997).

17. M. C. Romero Fuster, Sphere stratifications and the Gauss map. Proc. Royal Soc. Edinburgh, 95 A (1983), 115-136. MR 85c:58021

18. V. D. Sedykh, Some invariants of convex manifolds. Matemática Contemporânea 5 (1993), 187-198. MR 95j:57027

19. B. Smyth and F. Xavier, A sharp geometric estimate for the index of an umbilic on a smooth surface. Bull. London Math. Soc. 24 (1992), 176-180. MR 93b:53004

Instituto de Matemática e Estatistica, Universidade Federal de Goiás, 74001-970, Goî̂NIA, GO, BraziL

E-mail address: ragarcia@mat.ufg.br

Departamento de Matemática, Universidade Federal de São Carlos, 13560-905, São CARlos, SP, Brazil

E-mail address: dirce@dm.ufscar.br

Departamento de Geometria e Topologia, Universidad de Valencia, 46000, Valencia, SPAIN

E-mail address: romero@uv.es

Instituto de Ciências Matemáticas e de Computação, Universidade de São Paulo, Departamento de Matemática, Caixa Postal 668, 13560-970, São Carlos, SP, Brazil

E-mail address: maasruas@icmsc.sc.usp.br 